\title{
Is Viral Load Testing a Good Recommendation for Monitoring Health Outcomes of Patients on ART in Sub-Saharan Africa? An Economic Perspective
}

Felix Masiye*

Department of Economics, University of Zambia, Zambia

The rapid scale up of antiretroviral therapy (ART) for HIV patients in Sub-Saharan Africa is considered to epitomize the success of the global response to the HIV epidemic. As at 2013, about 7.5 million HIV patients were on antiretroviral therapy in SSA [1]. This number is growing due to increasing new infections, patients on ART living longer. In an effort to improve programme monitoring and patient outcomes, the WHO issued new guidelines in 2013 in which it is recommended that viral load testing HIV positive individuals are initiated in ART if their CD4 count is no more than 500, regardless of clinical symptoms [2]. In addition, countries have adopted the Option $\mathrm{B}+$ which requires that all $\mathrm{HIV}$-positive pregnant women are put on ART regardless of CD4 count, as well as increased access to testing will likely increase the number of people on ART. Clearly, as far as numbers go, the ART programme has been largely successful in reducing in adult and childhood mortality in Sub-Saharan Africa.

Despite this success, monitoring patients on treatment has proved suboptimal. As the global HIV/AIDS programme budget continues to escalate, concerns about quality of care and programme effectiveness in meeting its goals will become more paramount. Much of the evidence regarding the impact of ART on AIDS mortality, the ultimate health outcome measure, is largely circumstantial as in many countries in SubSaharan Africa direct measures of ART impact are missing. Here is what we know. Most programmes in Sub-Saharan Africa report fairly low patient retention levels even as early as 12 months following initiation onto ART. Studies have shown that patient attrition is quite high in many settings, in some cases as high as 35 percent following 24 months of ART [3]. Documentation about what happens to the considerable proportion of patients who are lost to follow up is even scarcer. Under such circumstances, assessing programme effectiveness and efficiency becomes difficult.

Part of this problem of suboptimal monitoring has been attributed to the inadequacies of the tools used to monitor patients, namely CD 4 count. Generally CD4 count at a point in time holds a poor relation to the effectiveness of ART on the health status of the patient. In this regard, high attrition rates negate the condition under which $\mathrm{CD} 4$ count would have been more robust in monitoring. In addition, even for patients on ART and in care, monitoring patient outcomes based on $\mathrm{CD} 4$ count has proved challenging. For example, poor sensitivity of $\mathrm{CD}$ 4 count to true underlying health status, results in as much as 30 percent of patients being unnecessarily switched from first line to much more expensive second line ART [4]. On the other hand, studies have shown that $\mathrm{CD} 4$ count has led to failure to detect failure of first line treatment causing death and disease progression. Under this patient monitoring regime, many patients may still die due to suboptimal treatment.

The third 90 in the recently announced UNAIDS 90:90:90 goal aims to achieve 90 percent of patients on treatment are responding successfully to their drugs, including viral load suppression, by 2020 [5]. Country examples have shown promise in using viral load testing with promising results. Achieving this goal will require scaling up viral load testing capabilities across countries in even resource-poor settings.
A case is made that the utility is CD count as a monitoring is hampered by the relatively low levels of adherence to routine testing in most parts of SSA. In 2013, the WHO has commended that HIV viral load testing be adopted, as viral load suppression is the most direct measure of ART response, and therefore HIV-associated mortality. With Dried Blood Spots-based HIV virological testing technology available, countries considering using Viral load testing on DBS samples which can be transported from health facilities to a central testing centres. However, viral load testing is enterprise is expensive and needs to be subjected to a cost effectiveness analysis.

On medical terms, a good case for HIV virological testing has been made. The potential economic consequences of better monitoring (e.g., costs of inappropriate switching patients to more expensive treatment and premature mortality due to suboptimal monitoring) are also well argued. However, there are concerns that these testing guidelines will put further strain on health systems which are already weak, thereby jeopardizing the ultimate goal of universal ART coverage. Critics have also argued that the resources being invested in viral load testing could be instead be devoted to further increasing the coverage of ART. In the face of limited resources, it is essential that a decision is based on a full evaluation which requires an economic appraisal in the form of costeffectiveness analysis is conducted.

\section{References}

1. UNAIDS (2013) Access to antiretroviral therapy in Africa status report on progress towards the 2015 targets. Geneva: UNAIDS.

2. WHO (2013) Consolidated guidelines on the use of antiretroviral drugs for treating and preventing HIV infection: Recommendations for a public health approach. WHO Geneva.

3. Fox MP, Rosen S (2010) Patient retention in antiretroviral therapy programs up to three years on treatment in sub-Saharan Africa, 2007-2009: systematic review. Tropical Medicine and International Health 1:1-15.

4. Sigaloff KCE, Hamers RL, Wallis CL, Kityo C, Siwale M, et al. (2011) Unnecessary antiretroviral treatment switches and accumulation of HIV resistance mutations; two arguments for viral load monitoring in Africa. J Acquir Immune Defic Syndr 1999 58: 23-31.

5. UNAIDS (2013) 90-90-90: An ambitious treatment target to help end the AIDS epidemic. Geneva: UNAIDS.

${ }^{*}$ Corresponding author: Felix Masiye, Department of Economics, University of Zambia, Zambia, Tel: +260 211293 348; E-mail: fmasiye@yahoo.com

Received December 01, 2015; Accepted December 02, 2015; Published December 06, 2015

Citation: Masiye F (2015) Is Viral Load Testing a Good Recommendation for Monitoring Health Outcomes of Patients on ART in Sub-Saharan Africa? An Economic Perspective. Health Econ Outcome Res Open Access 1: e102. doi: 10.4172/2471-268x/1000e102

Copyright: $\odot 2015$ Masiye F. This is an open-access article distributed under the terms of the Creative Commons Attribution License, which permits unrestricted use, distribution, and reproduction in any medium, provided the original author and source are credited 